\title{
Standardising Syndromic Classification in Animal Health Data
}

\author{
Fernanda C. Dórea*1, Céline Dupuy², Flavie Vial ${ }^{3}$, Crawford Revie $^{4}$ and Ann Lindberg ${ }^{1}$ \\ ${ }^{1}$ Department of Epidemiology and Disease Control, National Veterinary Institute, Uppsala, Sweden; ${ }^{2}$ French Agency for Food, \\ Environmental and Occupational Health \& Safety (ANSES), Lyon, France; 'Veterinary Public Health Institute, Bern, Switzerland; \\ ${ }^{4}$ Centre for Veterinary Epidemiological Research, Charlottetown, PE, Canada
}

\section{Objective}

To develop an ontology for the classification of animal health data into syndromes with application to syndromic surveillance.

\section{Introduction}

Veterinary syndromic surveillance (VSS) is a fast growing field, but development has been limited by the limited use of standards in recording animal health events and thus their categorization into syndromes. The adoption of syndromic classification standards would allow comparability of outputs from systems using a variety of animal health data sources (clinical data, laboratory tests, slaughterhouse records, rendering plants data, etc), in addition to improving the ability to compare outputs among countries.

The project "Standardising Syndromic Classification in Animal Health Data" (SSynCAHD) aims to standardize the classification of animal health records into syndromes.

\section{Methods}

SSynCAHD will make use of current technologies from information management, which aim to promote intelligent access to data. In particular, SSynCAHD will be informed by the latest thinking relating to the Semantic Web, which supports the development of frameworks to maximize the potential for data sharing and reuse.

The use of an ontology allows the knowledge base used for syndromic classification to be structured into a formal representation. Formally, "an ontology defines a common vocabulary for researchers who need to share information in a domain. It includes machineinterpretable definitions of basic concepts in the domain and relations among them" [1].

The use of ontologies in human syndromic surveillance has been previously explored by Conway and collaborators [2]. In animal health surveillance, further challenges are posed by the number of species monitored, the variety of data sources explored, and the lack of homogeneity in the syndromes defined in different systems.

In order to investigate the potential for developing an ontology for animal health syndromic classification, a workshop was held in May 2014, gathering interested experts with experience in veterinary syndromic surveillance and/or animal health data recording.

\section{Results}

The results of the workshop indicated strong interest in the field. It was proposed that development should be based on one syndrome at a time, and be data-driven; that is, collaborators would grow the ontology, for each syndrome, based on an analysis of the data sources available to them. The syndromes "reproductive" and "respiratory" were chosen as a starting point. The data-driven development will be intercalated with rounds of expert elicitation. Workshops and digital elicitation will serve to resolve conflicts, take decisions when needed, and ensure completeness of the concepts included in the ontology. The project will also promote the use of existing controlled vocabularies and classification standards in animal health. A follow-up workshop is planned for March 2015 in connection with the annual conference of the Society for Veterinary Epidemiology and Preventive Medicine.

The development work to be carried out prior to the 2015 workshop will consist of: an inventory of veterinary surveillance systems and the syndrome definitions used; an inventory of the use of ontologies in health surveillance; and development of a template of collaboration for the data-driven steps in ontology construction.

\section{Conclusions}

SSynCAHD proposes to harmonise syndromic surveillance data use rather than data recording. This will be achieved by standardising the classification of records into syndromes. Potential advantages include: an ability to achieve syndromic classification from different sources of data which are recorded using an institution's own vocabulary; the ability to compare VSS system outputs within and between countries; and the more timely development of VSS systems.

\section{Keywords}

Animal health; syndromic surveillance; ontology

\section{References}

[1] Ontology Development 101: A Guide to Creating Your First Ontology 2001. Available at http://protege.stanford.edu/publications/ontology_ development/ontology101.pdf.

[2] Conway M, Dowling J, Chapman W. Developing an application ontology for mining free text clinical reports: the extended syndromic surveillance ontology. In: 3rd international workshop on health document text mining and information analysis (LOUHI 2011); 2011. p. $75-82$.

\section{*Fernanda C. Dórea \\ E-mail: fernanda.dorea@sva.se}

